\title{
An Improved Scanning Strategy for Long Pulsed Laser Drilling of Carbon Fiber Textiles
}

\author{
Stefan Janssen, Markus Christian Eckstein \\ Fraunhofer Institute for Laser Technology ILT, Steinbachstr. 15, 52074 Aachen, Germany \\ stefan.janssen@ilt.fraunhofer.de
}

\begin{abstract}
Laser drilling of carbon fiber based textiles by means of long pulsed laser radiation and dynamic scanning optics offer great potential to increase the materialographical quality of drilling holes for force transmission elements. Compared to laser cutting or drilling of consolidated CFRP parts, thermally induced defects of the matrix do not occur when drilling textile preforms. By utilizing a scanner-guided laser drilling process for long pulsed fiber laser radiation with high pulse energy, the heat affected zone can be significantly large if heat accumulation effects are not taken into account. A pulse distribution strategy in order to avoid heat accumulation and to increase the materialographical quality while maintaining productivity is being presented and evaluated in this work.
\end{abstract}

DOI: 10.2961/jlmn.2018.01.0008

Keywords: drilling, CFRP, textile, preform, QCW fiber laser, scanning, HAZ, productivity

\section{Introduction}

Current state of the art of machining carbon fiber reinforced materials is to mechanically drill, cut or mill the work piece after the consolidation process. This can thus be regarded as rework after the consolidation process, e.g. a resin transfer molding (RTM) process. However, conventional mechanical processes lead to high tool wear and often to non-repairable defects in the work piece such as delamination, fiber pullout, cracks in matrix and fibers, and deformation. This is especially critical for the lifetime and performance during operation of the structural part. Hole diameters of a few millimeters are required for the integration of force transmission elements to apply loads to the carbon fiber structure. Ultra-sonic excited cutting knifes are not applicable in this case due to high aspect ratios of the hole. Punching as well as water jet cutting drop out due to force induced interaction with the textile or the necessity of a subsequent drying process.

Laser material processing has the potential to circumvent the above mentioned issues. By ultra-short pulsed laser processing it is possible to accurately process the carbon fiber preform with high geometrical precision and minimum defects. As investigated by Riveiro et al, the application of cw-laser sources results in more thermal damage to the workpiece than pulsed laser sources [1]. The cooling time during the pulsed operation mode allows the work piece to keep the overall thermal input at a significant lower level. The pulsed laser source induces less thermal damage with a decreasing duty cycle. Thus, becoming quasi-cw with high duty cycles prevents the workpiece from cooling down.

Fiber lasers with high pulse energy have the potential to increase productivity but relate to laser-induced materialographical quality issues. This happens due to heat accumulation caused by laser radiation when the carbon fiber structure has not enough time to cool down before the subsequent laser pulse interacts. The extent of the resulting heat affected zone (HAZ) is crucial for the mechanical properties of the bond with the insert. Some of the mechanisms responsible for the formation of the HAZ were already assessed by previous works. Negarestani et al. investigated several process parameters such as pulse frequency, pulse energy and scanning speed with a nano-second pulsed laser [2]. They found a relationship between the repetition rate and pulse energy with the fiber pull out in the top and bottom kerf as their main quality benchmark. In addition, the use of inert nitrogen gas with some parts of oxygen at a pressure of 8 bars increased the process quality. This is due to less exothermic reactions as observed with ambient gas or pure oxygen as also investigated by Sato et al. [3]. They both agreed on the fact that thermal conduction is crucial for a decreased HAZ. However, some parts of oxygen accelerate the cutting process with an increased material removal rate.

Evaluating the extent of the HAZ is therefore crucial for the development of an advanced process strategy. Currently, there is no standardized way of measuring the HAZ for carbon fiber preforms. Only the properties essential for the production process and the component functionality are being evaluated. These are thermal damages to the surface of the carbon fiber preform and the sizing [4]. The sizing is applied on the carbon fibers before winding them on a roving. The main functionality of the sizing is to increase the adhesion between the carbon fibers with the matrix. In addition, the sizing serves as a protection layer for the carbon fiber surface and decreases the friction between the single carbon fiber filaments. The friction coefficient between carbon fibers and tool machine parts is also reduced as well as the electrostatic charge. Therefore, it is pivotal to secure the retention of the sizing on carbon fibers. To evaluate the damage of the sizing, the back-burn of the knitting yarn is measured as means to evaluate the HAZ. This is due to the similar melting points of the sizing $\left(\mathrm{T} \approx 250^{\circ} \mathrm{C}\right)$ and the knitting yarn $\left(\mathrm{T} \approx 260^{\circ} \mathrm{C}\right)$. In addition, the mechanical properties of carbon fibers start being influenced at a temperature of $\mathrm{T} \approx 300^{\circ} \mathrm{C}$. [5] 
Previous works on the thermal damage of CFRP composites instead evaluated the HAZ formation by subdividing into matrix evaporation zone (MEZ) and resin alteration zone (RAZ) [3]. Since in this work no resin is used due to the preform character, this subdivision cannot be applied and the thermal damage to the resin at lower temperatures is no issue as described in Riveiro et al. [1].

With the thermal damage to the material as the main threat when processing the preform with a laser source, an advance in the processing technique is an option of increasing the part quality. As already shown with processing semiconductor wafers with laser sources, an advance in the processing technique leads to improved results. However, in the mentioned example, the cut still is made with multiple scans instead of one-shot penetration, it as a first approach to incorporate an advanced scanning strategy with already developed parameters to cut the work piece efficiently. [6]

In addition, the process time is crucial for high scale production to remain profitable. As for now, $50 \%$ of the costs of a CFRP structures are due to the production process. The other $50 \%$ are accounted for the preform itself and the matrix. Machining and tool costs take $30 \%$ of the overall costs of a carbon fiber structure [7]. Thus, reducing the process time and using wearless tools such as fiber laser radiation will have a significant impact on reducing the overall costs of the production process of carbon fiber structures.

\section{Experimental Setup}

Within this work, non-crimp carbon fiber preforms with thicknesses of $\sim 3 \mathrm{~mm}$ (5 bi-axial layers) are investigated regarding laser processing by means of long pulsed fiber laser radiation. The influence of different process parameters is being evaluated regarding the HAZ and appearance of a solid layer at the hole wall. The temperature distribution during processing is important for estimating when and where the knitting yarn, which keeps the carbon fiber plies together, disintegrates. Without the knitting yarn not only preform swelling but also shifting of textile layers might occur which is not desired for subsequent process steps in the production chain of CFRP components.

The laser source used in the scope of this work is an IPG Photonics YLS-600/6000-QCW fiber laser with a maximum pulse peak power of $6 \mathrm{~kW}$ and $600 \mathrm{~W}$ average power. The technical specifications of the fiber laser source and the connected scanning system are listed in the table below.
Table 1 Technical specifications of the QCW fiber laser source and the scanning system

IPG YLS-600-6000 QCW-AC

(QCW laser beam source)

\begin{tabular}{ll}
\hline Average Power & $\max .600 \mathrm{~W}$ \\
Pulse Peak Power & $\max .6 \mathrm{~kW}$ \\
Pulse Duration & $0.2-10 \mathrm{~ms}$ \\
Repetition Rate & $10-500 \mathrm{~Hz}$ \\
Beam Quality $\left(\mathrm{M}^{2}\right)$ & 5.87 \\
Wavelength & $1070 \mathrm{~nm}$ \\
Process fiber diameter & $100 \mu \mathrm{m}$ \\
\hline
\end{tabular}

ScanLab IntelliScan III 30

(Galvo scanner with collimated telecentric optics)

Beam Aperture $\max .30 \mathrm{~mm}$

Marking Speed $\max .0 .7 \mathrm{~m} / \mathrm{s}$

Positioning Speed $\max .9 .0 \mathrm{~m} / \mathrm{s}$

Scan Field $\quad 100 \times 100 \mathrm{~mm}$

Focal Length $163 \mathrm{~mm}$

Focal Diameter $\quad 82 \mu \mathrm{m}$

Collimation length $\quad 200 \mathrm{~mm}$

Optical transmissivity (entire system) $\quad 85 \%$

Especially the positioning speed of the scanner is a crucial factor in the scope of the experiments conducted in this work. The real maximum positioning speed has been measured to be $8.2 \mathrm{~m} / \mathrm{s}$ for the strategy developed in this work. The utilized scanner software is Scan2D.

\subsection{Materials \& Preparation}

The investigated material is a carbon fiber based dry preform consisting of multiple plies of bi-axial $\left( \pm 45^{\circ}\right)$ noncrimp fiber layers with a mass per unit area of $410 \mathrm{~g} / \mathrm{m}^{2}$ for each bi-axial ply. For clarification it should be mentioned that one layer itself consists of two plies $\left(+45^{\circ} / 45^{\circ}\right)$ which are held together by a knitting yarn. The knitting yarn consists of PES (Polyethersulfone) which has a similar melting point to the sizing of the fiber. The carbon fiber is a $50 \mathrm{k}$ filament HT-fiber (High Tenacity), type Panex 35, from Zoltek Inc.. Thicker preforms are produced by stacking up several bi-axial layers upon each other. No binder is used to additionally fix several stacked bi-axial layers.

During laser drilling the preform is encapsulated in a closed process chamber which has a window on the top for letting through laser radiation. A suction system is applied to the chamber for removing dust, loose fibers and smoke. During the laser material processing of dry carbon fiber textiles, particles are ejected from the laser-material interaction zone. One side of the chamber has a port for connecting the suction system. On the opposite side is an inlet for ventilation. The bottom plate is an aluminum plate with screw holes for fixation on an $x-y$ handling system.

The purpose of the conducted experiments is to determine the process window for productively manufacturing notches in a multi-layer non-crimp CFRP-preform with minimum HAZ but high productivity for long pulse laser drilling via scanning optics. 


\subsection{Scanning strategy for long pulsed laser drilling}

\section{Preconditions}

The main research topic in utilizing long pulse laser radiation for drilling carbon fiber based preforms is to manufacture geometrical and materialographical high quality holes with precise entry and exit diameters as well as achieving a small heat affected zone around the hole. Due to high pulse energies of up to several Joule it is possible to fully penetrate stacked carbon fiber preforms with several millimeter thickness with one pulse. Penetrating the fiber material with one pulse is required for the scanning strategy proposed in this work. If the multilayer carbon fiber preform cannot be penetrated within one laser pulse but requires several pulses per spot (percussion drilling) the pressure generated in the holes leads to uncontrolled fiber movement after each pulse [8]. Hence, the internal structure of the preform can change in terms of deflection of fiber orientation und generation of cavities. On the other hand the pulse energy should be small enough not to induce unnecessary heat which would lead to excessive burning of the sizing which covers the carbon fibers. Removing the sizing leads to less adhesion forces between the fibers and the matrix resulting in lower maximum transmission forces.

In the end of the process a continuously cut hole contour is required in order to integrate force transmission elements. Thus, a sufficient overlap of the pulses is required to achieve an entirely cut hole. If the pulse overlap is very high the productivity can be negatively influenced. The pulse overlap based on DIN 32540 is depicted in Fig. 1 [9].

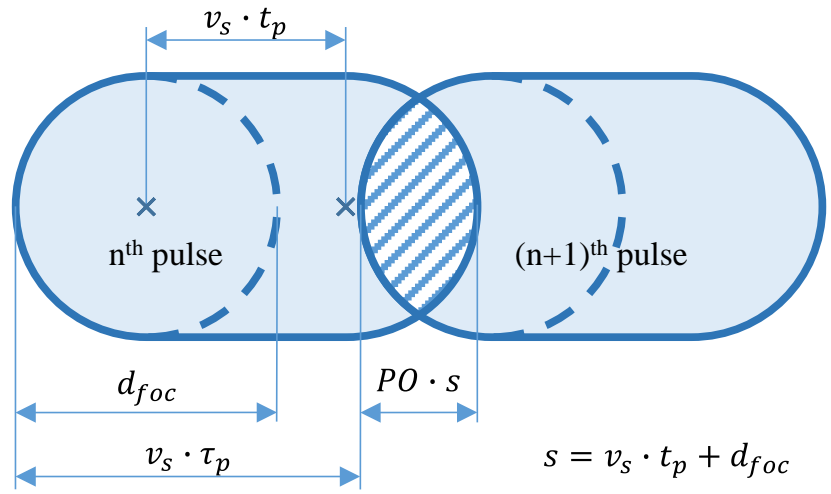

Fig. 1 Pulse overlap of moving pulsed laser radiation

Whereas $v_{s}$ is the scanning speed, $t_{p}$ the pulse duration, $d_{f o c}$ the focal diameter, $\tau_{p}$ the periodic time and $P O$ the pulse overlap. In the scope of this work a point-andshoot method is being used. Thus, $v_{s} \cdot t_{p}=0$ and the scan- ning speed $v_{s}$ becomes the jump speed $v_{j}$ which has been measured as $8.2 \mathrm{~m} / \mathrm{s}$. A conventional scanning process for manufacturing a continuous cut can schematically be depicted as shown in Fig. 3.

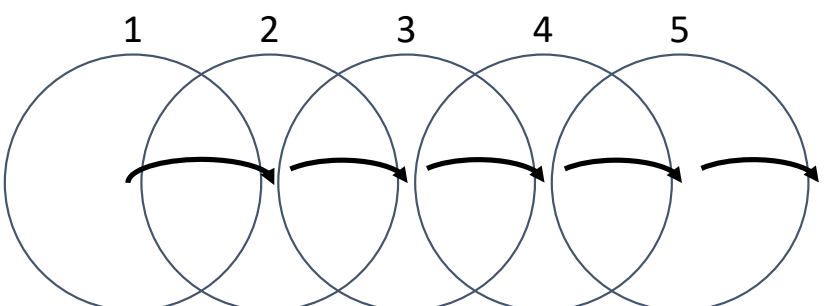

Fig. 3 Schematic picture of conventional scanning with pulsed laser radiation

Carbon fibers are highly electrical and heat conductive. At the same time, carbon fibers have a high specific heat capacity. Due to the rapid loss of energy via heat conduction from the laser-material interaction area, not only the HAZ expands but also the structure of the carbon fibers at the fiber ends at the hole wall changes. If the pulse energy is too high or the cooling time between pulses is too low a significant hard and brittle layer appears at the hole wall which can negatively influences further processing of the preform [10]. Hence Table 2 describes the potential benefits and drawbacks for long pulse laser drilling of preforms in terms of repetition rate and pulse overlap.

Table 2 Main advantages and disadvantages for relevant process parameter combinations during laser drilling by means of a conventional scanning process

\begin{tabular}{lll}
\hline $\begin{array}{l}\text { Process } \\
\text { parameter }\end{array}$ & $\begin{array}{c}\text { Main } \\
\text { advantage (+) }\end{array}$ & $\begin{array}{c}\text { Main } \\
\text { disadvantage (-) }\end{array}$ \\
$\begin{array}{l}\text { Repetition rate } \uparrow \\
\text { complete cut }\end{array}$ & $\begin{array}{l}\text { coverlap } \uparrow \\
\text { mulation } \rightarrow \text { HAZ }\end{array}$ \\
\hline $\begin{array}{l}\text { Repetition rate } \uparrow \\
\text { Pulse overlap } \downarrow\end{array}$ & $\begin{array}{l}\text { small process } \\
\text { time } \rightarrow \text { high } \\
\text { productivity }\end{array}$ & $\begin{array}{l}\text { risk of incom- } \\
\text { plete cut } \\
\text { high heat accu- } \\
\text { mulation } \rightarrow \text { HAZ }\end{array}$ \\
\hline $\begin{array}{l}\text { Repetition rate } \downarrow \\
\text { Pulse overlap } \uparrow\end{array}$ & $\begin{array}{l}\text { small HAZ } \\
\text { complete cut }\end{array}$ & $\begin{array}{l}\text { high process time } \\
\text { tivall produc- } \\
\text { tivity }\end{array}$ \\
\hline $\begin{array}{l}\text { Repetition rate } \downarrow \\
\text { Pulse overlap } \downarrow\end{array}$ & small HAZ & $\begin{array}{l}\text { risk of incom- } \\
\text { plete cut } \\
\text { high process time }\end{array}$ \\
\hline
\end{tabular}

As a result from Table 2, it is not possible to simultaneously achieve a reproducible, productive drilling process with a small HAZ via conventional scanning. An approach

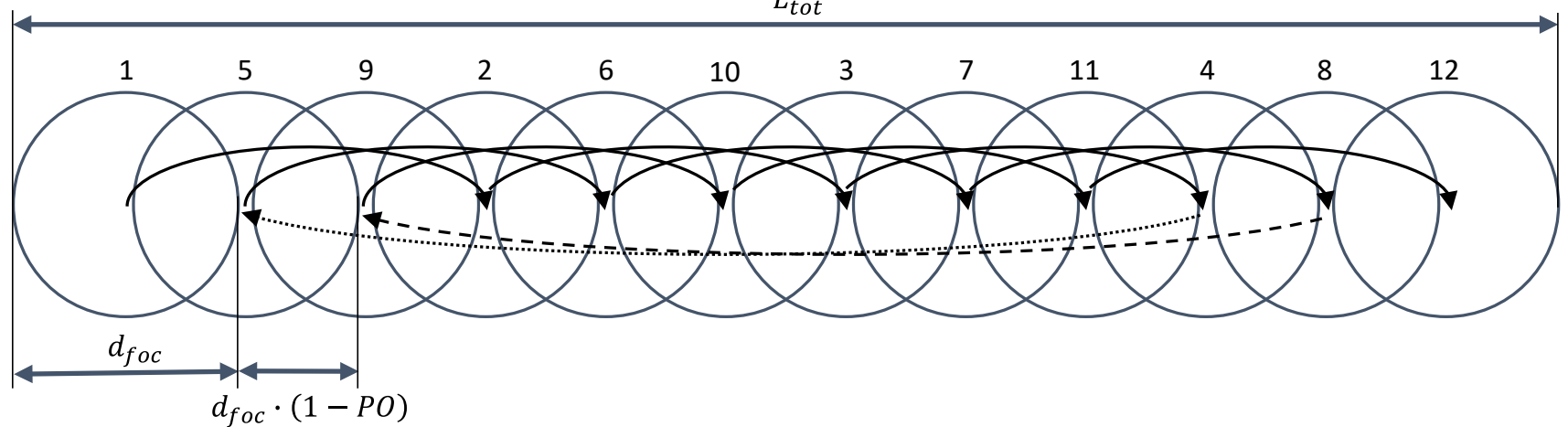

Fig. 2 Schematic example of an alternative scanning strategy for high productive and high quality manufacturing of cuts in carbon fiber preforms 
for an alternative scanning process is to distribute the pulses over the total scanning contour in a way that the geometrical distance between each two subsequent pulses is significantly increased. In this case, heat accumulation due to pulse-to-pulse interaction does not occur. Additionally, the final pulse overlap between each directly neighboring pulses should be sufficient to achieve a reproducible complete cut in the preform. Derived from these conditions, Fig. 2 schematically depicts the proposed pulse distribution strategy for a high productive scanning process with small HAZ.

The total length of the cutting contour $L_{\text {tot }}$ is fixed if the size of the force transmission element is known. In this case, the minimum required amount of pulses $N_{P \text {,min }}$ depending on the total length, the focal diameter $d_{f o c}$ and a predetermined pulse overlap $P O$ is:

$$
N_{P, \text { min }}=\left\lceil\frac{L_{t o t}-d_{f o c} \cdot P O}{d_{f o c} \cdot(1-P O)}\right\rceil
$$

Having the minimum amount of pulses the minimum required process time $t_{\text {proc,min }}$ can be calculated:

$$
t_{\text {proc, } \min }=\frac{N_{P, \text { min }}}{f_{\text {laser }}}
$$

in which $f_{\text {laser }}$ is the repetition rate of the laser source. Now having the possibility to distribute pulses over the entire contour length of the hole or cut, a time delay $\Delta t_{\text {virtual }}$ between two directly neighboring and overlapping pulses (e.g. pulse no. 1 and 5, Fig. 2) can be set. The inverse of this time delay will be a virtual repetition rate $f_{\text {virtual }}$ between each directly neighboring pulsed which should be significantly lower than the repetition rate of the laser $f_{\text {Laser }}$.

Every pulse within any pass of pulses (e.g. pulses no. 14, 5-8 or 9-12, Fig. 2) have an equal distance. Each pass or line is only shifted by a distance $d_{f o c} \cdot(1-P O)$ due to the constant pulse overlap. Knowing the minimum required process time $t_{\text {proc,min }}$ and having chosen an initial value for the virtual repetition rate $f_{\text {virtual }}$, a number of lines $N_{L}$ can be calculated:

$$
N_{L}=\left\lceil t_{\text {proc, min }} \cdot f_{\text {virtual }}\right\rceil
$$

The number of pulses per line $N_{P / L}$ can be calculated in two ways: either by using the minimum number of pulses $N_{P \text {,min }}$ and the number of lines $N_{L}$ or by calculating the ratio of the repetition rate of the laser $f_{\text {laser }}$ and the virtual repetition rate $f_{\text {virtual }}$ :

$$
N_{P / L}=\left\lceil\frac{N_{P, \text { min }}}{N_{L}}\right\rceil \vee N_{P / L}=\left\lceil\frac{f_{\text {Laser }}}{f_{\text {virtual }}}\right\rceil
$$

If the ratio of $f_{\text {Laser }}$ and $f_{\text {virtual }}$ is a natural number both calculations of $N_{P / L}$ lead to the same solution. If the solutions are not equal, the true virtual repetition rate $f_{\text {virtual,true }}$ changes to:

$$
f_{\text {virtual,true }}=\frac{f_{\text {laser }}}{N_{P / L}}
$$

Due to several ceiling functions to get integer values the true number of pulses can now be calculated:

$$
N_{P}=N_{P / L} \cdot N_{L}=t_{\text {proc }} \cdot f_{\text {Laser }}
$$

Since $N_{P \text {,min }}$ and $N_{P}$ are not necessarily equal the true pulse overlap $P O_{\text {true }}$ can now be calculated:

$$
P O_{\text {true }}=1-\frac{L_{\text {tot }}-d_{f o c}}{d_{f o c} \cdot\left(N_{P}-1\right)}
$$

The displacement $d s$ between two subsequent overlapping lines can be calculated with the true pulse overlap:

$$
d s=d_{f o c} \cdot\left(1-P O_{\text {true }}\right)
$$

The parameters from equation (1) to (8) are sufficient to set up the proposed improved scanning strategy. There are a few more informative parameters which have significant impact on the achievable quality in terms of HAZ and productivity. One is the distance $\Delta s$ between two pulses within one line which is important for the heat accumula-

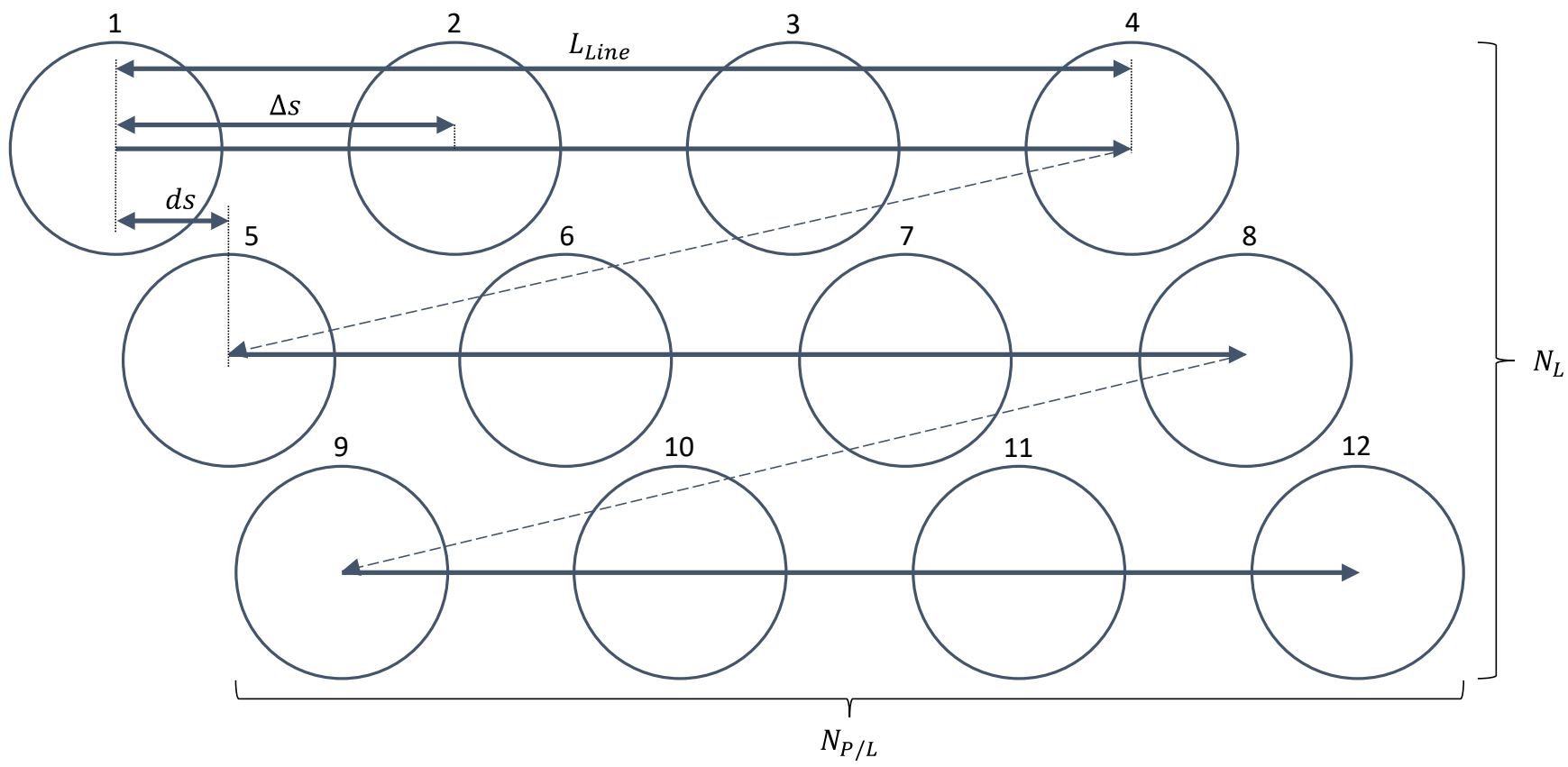

Fig. 4 Schematic picture of the pulse distribution scanning strategy decomposed into individual lines 
tion induced by the heat flow along the carbon fiber:

$$
\Delta s=d_{\text {foc }} \cdot\left(1-P O_{\text {true }}\right) \cdot N_{L}
$$

The geometrical distance $\Delta s$ should be large enough so that heat accumulation along the way between each two holes in one line is prevented as much as possible. Fig. 4 schematically shows the pulse distribution strategy decomposed into individual lines for an exemplary twelve pulses cutting process. The number above each circle is the pulse number.

Heat accumulation in this scan strategy can thus appear due to two main factors:

1. Heat accumulation due to insufficient cooling time between two overlapping pulses of each two subsequent lines (e.g. pulses 1 and 5; temporal pulseto-pulse or - in this case - scan-to-scan heat accumulation)

2. Heat accumulation due to insufficient time and distance between two pulses within one line (e.g. pulses 1 and 2; temporal-spatial pulse-to-pulse heat accumulation)

The true total process time $t_{\text {proc,true }}$ can easily be calculated by dividing the true total number of pulses $N_{P}$ by the repetition rate of the laser $f_{\text {Laser }}$. In the case of a straight line, the maximum repetition rate of the laser is mainly limited by the maximum jumping distance from the end of one line back to the beginning of the subsequent line $L_{\text {Line }}$ :

$$
L_{\text {Line }}=d_{\text {foc }} \cdot\left(1-P O_{\text {true }}\right) \cdot N_{L} \cdot\left(N_{P / L}-1\right)
$$

The required jump time $t_{\text {jump }}$ is thus the ratio of the length of one line $L_{\text {Line }}$ and the jump speed of the scanner $v_{\text {jump }}$ :

$$
t_{\text {jump }}=\frac{L_{\text {Line }}-d_{f o c} \cdot\left(1-P O_{\text {true }}\right)}{v_{\text {jump }}}
$$

The maximum repetition rate $f_{\text {Laser,max }}$ of the laser is thus bound to the pulse duration $t_{p}$, the maximum jump speed of the scanner and potential scanner delays $t_{\text {delay }}$ set in the scanning software:

$$
\frac{1}{f_{\text {Laser,max }}}-t_{p}-t_{\text {delay }}-t_{\text {jump }} \leq 0
$$

The necessary time for acceleration and deceleration of the galvanometer mirrors has been neglected in equation 12 .

Now all important parameters have been derived for implementing a pulse distribution strategy which leads to a productive scanning process due to an overall high repetition rate of the laser and simultaneously to a significant smaller HAZ due to low local pulse-to-pulse heat accumulation.

The critical factors that have to be determined experimentally for a given cutting length $L_{t o t}$ are:

a. Minimum required pulse energy $E_{P \text {, min }}$ for one shot penetration

b. Minimum required pulse overlap $P O_{\min }$ for making a continuous cut c. Virtual repetition rate $f_{\text {virtual }}$ for determining the smallest HAZ without temporal pulse-to-pulse heat accumulation

d. Distance between pulses in one line $\Delta s$ for determining the minimum distance to prevent spatial pulse-to-pulse heat accumulation

The laser repetition rate and the distance between pulses in one line are interdependent. For a good approximation of the relationship between $\Delta s$ and $f_{\text {Laser }}$ all ceiling functions in the equations (1) to (4) can be neglected which will lead to:

$$
\Delta s \approx \frac{\left(L_{\text {tot }}-d_{f o c} \cdot P O_{\text {min }}\right)}{f_{\text {Laser }} \cdot f_{\text {virtual }}^{-1}} \stackrel{L_{\text {tot }} \gg d_{\text {foc }}}{\approx} \frac{L_{\text {tot }} \cdot f_{\text {virtual }}}{f_{\text {Laser }}}
$$

After having determined the maximum allowed repetition rate $f_{\text {Laser }}$ without significant heat accumulation the total process time can be calculated analogically to equation (2).

In order to test the pulse distribution strategy, a flat five bi-axial layer carbon fiber preform is being cut over a total length $L_{t o t}$ of $30 \mathrm{~mm}$. The cut is being investigated regarding the necessary pulse energy for one-shot penetration, required pulse overlap for a continuous cut without any uncut fibers and the size of HAZ.

\section{Results and Discussion}

\section{Required pulse energy for one-shot penetration}

As a result from preliminary tests, one shot penetration was safely possible with pulse energies bigger than $2 \mathrm{~J}$ and was not possible with pulse energies smaller than $0.8 \mathrm{~J}$ per pulse. In order to include the impact of pulse duration and pulse peak power separately, the process parameter sheet depicted in Table 3 was carried out with single pulse drill-

\begin{tabular}{|c|c|c|c|c|c|c|c|}
\hline & \multicolumn{6}{|c|}{ (Set) Pulse peak power [kW] } \\
\hline & & 1 & 2 & 3 & 4 & 5 & 6 \\
\hline \multirow{9}{*}{ 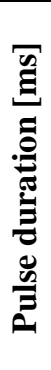 } & 0.2 & 0.17 & 0.34 & 0.51 & 0.68 & 0.85 & 1.02 \\
\hline & 0.3 & 0.255 & 0.51 & 0.765 & 1.02 & 1.275 & 1.53 \\
\hline & 0.4 & 0.34 & 0.68 & 1.02 & 1.36 & 1.7 & 2.04 \\
\hline & 0.5 & 0.425 & 0.85 & 1.275 & 1.7 & 2.125 & 2.55 \\
\hline & 0.6 & 0.51 & 1.02 & 1.53 & 2.04 & 2.55 & 3.06 \\
\hline & 0.7 & 0.595 & 1.19 & 1.785 & 2.38 & 2.975 & 3.57 \\
\hline & 0.8 & 0.68 & 1.36 & 2.04 & 2.72 & 3.40 & 4.08 \\
\hline & 0.9 & 0.765 & 1.53 & 2.295 & 3.06 & 3.825 & 4.59 \\
\hline & 1.0 & 0.85 & 1.70 & 2.55 & & 4.25 & 5.10 \\
\hline
\end{tabular}
ing in a five bi-axial layer preform.

Table 3 Investigated pulse energy in $[\mathrm{J}]$ for the determination of the minimum required pulse energy (green: through, orange: partly through, red: not through)

The preform was penetrated and marked as through if the backlight from a microscope was clearly visible through the hole. Each parameter was carried out three times. Some combinations have the same pulse energy and therefore lead to higher counting bars in Fig. 5. 


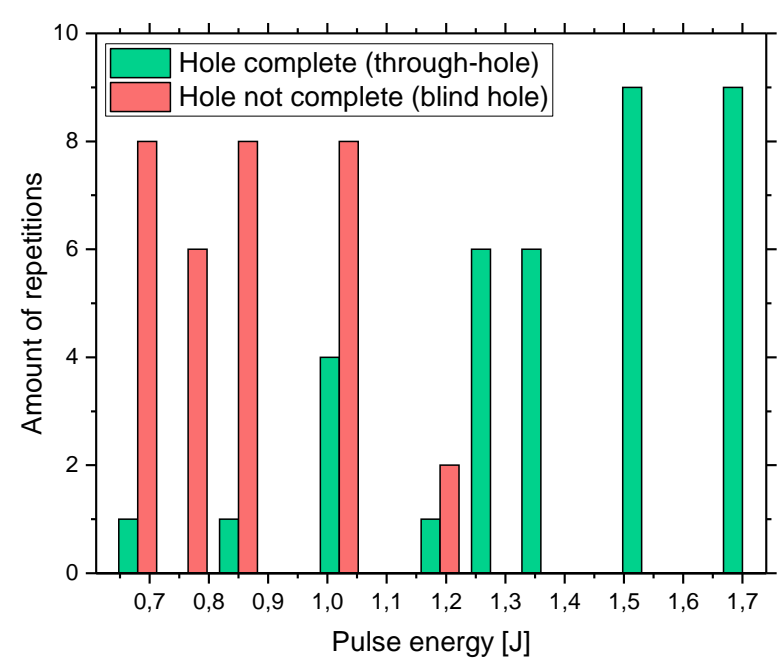

Fig. 5 Determination of the minimum required pulse energy (optical transmissivity included) for one shot penetration of five bi-axial layered carbon fiber preforms

Hence, the minimum pulse energy $E_{P, \min }$ for one shot penetration of five bi-axial layered carbon fiber preforms is about $1.275 \mathrm{~J}$. It should be mentioned that the hole diameters for holes drilled with $1.275 \mathrm{~J}$ are about $133 \mu \mathrm{m}$ for the entry and $125 \mu \mathrm{m}$ for the exit. Hence, the hole diameter is naturally larger than the focal diameter due to the significant energy input and, thus, heat flow along and across the fiber. This means that in all equations $d_{f o c}$ could be replaced by the actual exit hole diameter $d_{\text {hole }}$. Since this can be regarded as a measure for improving the process time, the theoretical diameter $d_{f o c}$ will be used in the course of this work.

Required pulse overlap

The minimum required pulse overlap can be determined with aid of conventional scanning tests with a fixed repetition rate and variation of the scanning speed over a length of $30 \mathrm{~mm}$. The preform is evaluated as safely and reproducibly cut if no uncut fibers across the cutting kerf remain. Since the scanning speeds are relatively low the simplified formula for the pulse overlap will be used:

$$
P O_{\text {simple }}=1-\frac{v_{\text {scan }}}{d_{f o c} \cdot f_{\text {Laser }}}
$$

The process parameter sheet and the results for different pulse overlaps are shown in Table 4. The laser repetition rate is $10 \mathrm{~Hz}$.
Table 4 Investigated pulse overlaps for a continuous scan cut (green: safely and reproducibly cut; yellow: few uncut fibers across the cutting kerf; red: significant number of uncut fibers)

\begin{tabular}{ccrc}
$\begin{array}{c}\text { Pulse over- } \\
\text { lap [\%] }\end{array}$ & $\begin{array}{l}\text { Scanning } \\
\text { velocity [mm/s] }\end{array}$ & $\begin{array}{r}\text { Process } \\
\text { time [s] }\end{array}$ & $\begin{array}{l}\text { Number } \\
\text { pulses per spot }\end{array}$ \\
\hline 0 & 0.81 & 37.0 & 1 \\
10 & 0.73 & 41.1 & $1-2$ \\
20 & 0.65 & 46.1 & $1-2$ \\
30 & 0.57 & 52.6 & $1-2$ \\
40 & 0.49 & 61.2 & $1-2$ \\
50 & 0.41 & 73.2 & 2 \\
60 & 0.32 & 93.7 & $2-3$ \\
70 & 0.24 & 125.0 & $3-4$ \\
78 & 0.18 & 187.5 & 5 \\
\hline
\end{tabular}

Starting from $50 \%$ pulse overlap no uncut fibers remain in the cutting kerf. By the number of pulses per spot it is shown how often any point on the scanning patch is being hit by laser pulses. In theory, $0 \%$ pulse overlap could be sufficient because each focal diameter connects with the previous one (Fig. 6).

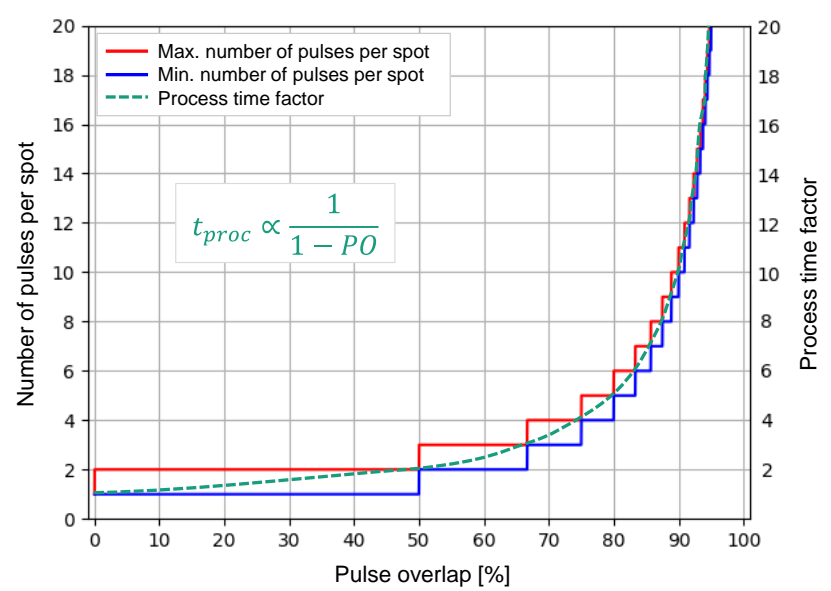

Fig. 6 Minimum, maximum number of pulses per spot and process time factor depending on pulse overlap

Due to the fact that the fibers are not fixed in position but are movable to a certain extent, a bigger pulse overlap and, thus, a bigger number of pulses per spot is required for laser cutting without leaving any uncut fibers in the cutting kerf. It can now easily be seen that increasing the pulse overlap not only increases the chances of cutting all fibers in the cutting kerf but can also significantly increase the process time, especially at high overlap values.

Determination of a suitable virtual repetition rate as an indicator for the minimum achievable HAZ

Several lines, each with a length of $30 \mathrm{~mm}$, are scanned continuously with a linear pulse overlap of about $50 \%$. The scanning velocity is varied in order to evaluate the HAZ for various laser repetition rates. The minimum scanning speed is $0.18 \mathrm{~mm} / \mathrm{s}$ due to scanner related soft- and hardware limitations. That is why repetition rates starting from $5 \mathrm{~Hz}$ are investigated (Table 5). The experiments are designed in a way that each parameter set both has a similar pulse overlap and total emitted energy $E_{\text {tot }}$ from the 
laser source. By this design, the expansion of the HAZ due to temporal pulse-to-pulse heat accumulation can be identified. There is a waiting time of two minutes between each experiment to let the preform cool down.

Table 5 Investigated laser repetition rates for continuously scanned cuts

\begin{tabular}{|c|c|c|c|c|}
\hline $\begin{array}{l}\text { Laser rep- } \\
\text { etition rate } \\
f_{\text {Laser }} \\
{[\mathrm{Hz}]}\end{array}$ & $\begin{array}{l}\text { Scanning } \\
\text { velocity } \\
v_{\text {scan }} \\
{[\mathrm{mm} / \mathrm{s}]}\end{array}$ & $\begin{array}{l}\text { Process } \\
\text { time } \\
t_{\text {proc }} \\
{[\mathrm{s}]}\end{array}$ & $\begin{array}{l}\text { Total } \\
\text { emitted } \\
\text { energy } \\
E_{\text {tot }}[\mathrm{J}]\end{array}$ & $\begin{array}{l}\text { Pulse } \\
\text { overlap } \\
P O_{\text {simple }} \\
{[\%]}\end{array}$ \\
\hline 5 & 0.20 & 150.00 & 1125.00 & 50.92 \\
\hline 10 & 0.41 & 73.17 & 1097.56 & 49.69 \\
\hline 15 & 0.61 & 49.18 & 1106.56 & 50.10 \\
\hline 20 & 0.81 & 37.04 & 1111.11 & 50.31 \\
\hline 30 & 1.22 & 24.59 & 1106.56 & 50.10 \\
\hline 40 & 1.63 & 18.40 & 1104.29 & 50.00 \\
\hline 50 & 2.04 & 14.71 & 1102.94 & 49.94 \\
\hline 60 & 2.44 & 12.30 & 1106.56 & 50.10 \\
\hline 70 & 2.85 & 10.53 & 1105.26 & 50.04 \\
\hline 100 & 4.07 & 7.37 & 1105.65 & 50.06 \\
\hline 150 & 6.11 & 4.91 & 1104.75 & 50.02 \\
\hline 200 & 8.15 & 3.68 & 1104.29 & 50.00 \\
\hline
\end{tabular}

The size of the HAZ for different laser repetition rates is shown in Fig. 7. Pulse-to-pulse heat accumulation already takes place at a laser repetition rate of $5 \mathrm{~Hz}$ and constantly increases up to about $40 \mathrm{~Hz}$. Afterwards, the HAZ on the exit side remains relatively constant considering the statistical deviation. The HAZ on the entry side is always smaller than the exit. It follows the same trend as the HAZ on the exit but becomes almost as big at a repetition rate of $200 \mathrm{~Hz}$. Up to $40 \mathrm{~Hz}$, which corresponds to an average laser power of $51 \mathrm{~W}$, convection cooling during the laseroff times limits the expansion of the HAZ on the carbon fiber preform. For higher repetition rates, resp. higher power, convection cooling during the pulse pauses is insufficient and the HAZ increases to its maximum size of about $7.5 \mathrm{~mm}$ in average. The inflections of the HAZ size between $40 \mathrm{~Hz}$ and $200 \mathrm{~Hz}$ are due to inhomogeneous layer contact within and in between bi-axial carbon fiber layers which lead to increased statistical deviations at higher repetition rates.

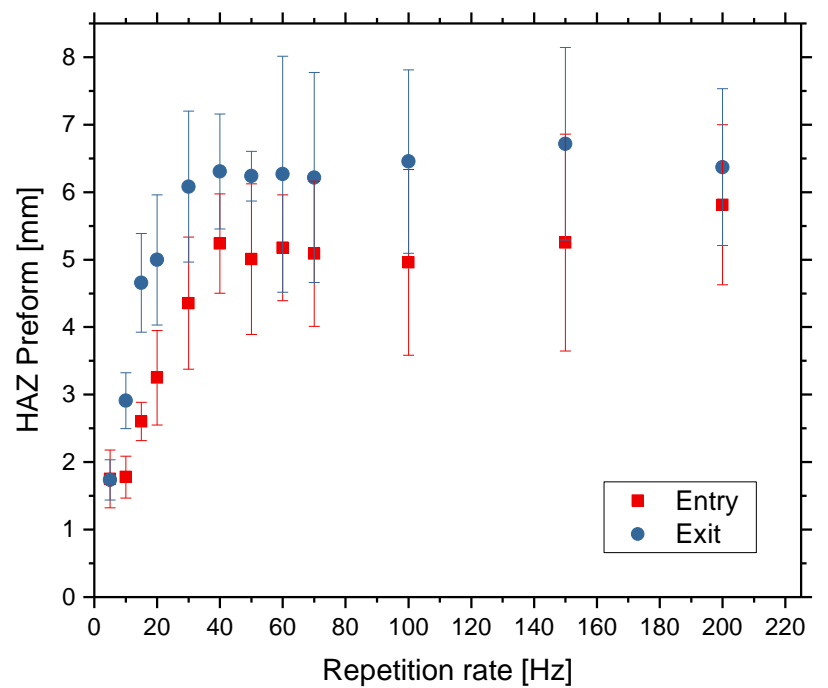

Fig. 7 Heat affected zone for preforms depending on the laser repetition rate

As a result, the minimum damage due to heat accumulation can be found for laser repetition rates in the range of a few Hertz where the influence of convection cooling is most effective. In order to determine the last parameter for the pulse distribution strategy a virtual repetition rate of $5 \mathrm{~Hz}$ is chosen.

Determination of the distance between pulses in one line as an indicator for temporal-spatial pulse-to-pulse heat accumulation

Having determined a virtual repetition rate of $5 \mathrm{~Hz}$, the productivity is meant to be increased by increasing the laser repetition rate $f_{\text {Laser }}$. By varying the laser repetition rate for a given total cutting length $L_{t o t}$ the distance between pulses in one line changes as shown in equation 13. In order to determine the minimum required distance, different total cutting lengths between $4 \mathrm{~mm}$ and $80 \mathrm{~mm}$ are investigated.

Table 6 Investigated distance between pulses in one line depending on the laser repetition rate and the total cutting length

\begin{tabular}{lll}
$\begin{array}{l}\text { Repetition } \\
\text { rate } f_{\text {Laser }} \\
{[\mathrm{Hz}]}\end{array}$ & $\begin{array}{l}\text { Total length } L_{\text {tot }} \\
{[\mathrm{mm}]}\end{array}$ & $\begin{array}{l}\text { Corresponding dis- } \\
\text { tance between pulses } \\
\text { in one line } \Delta s[\mathrm{~mm}]\end{array}$ \\
\hline 10 & $4-20(\Delta 4) ; 40 ; 80$ & $2-10(\Delta 2) ; 20 ; 40$ \\
20 & $8-40(\Delta 8) ; 80$ & $2-10(\Delta 2) ; 20$ \\
50 & $20-80(\Delta 20)$ & $2-8(\Delta 2)$ \\
70 & $28 ; 56 ; 84$ & $2 ; 4 ; 6$ \\
100 & $40 ; 80$ & $2 ; 4$
\end{tabular}

For laser repetition rates of $10 \mathrm{~Hz}$ the $\mathrm{HAZ}$ does not significantly increase even if the distance between pulses is as short as a few millimeters (Fig. 8). For $20 \mathrm{~Hz}$, the HAZ increases at distances of about $6 \mathrm{~mm}$ and less. 


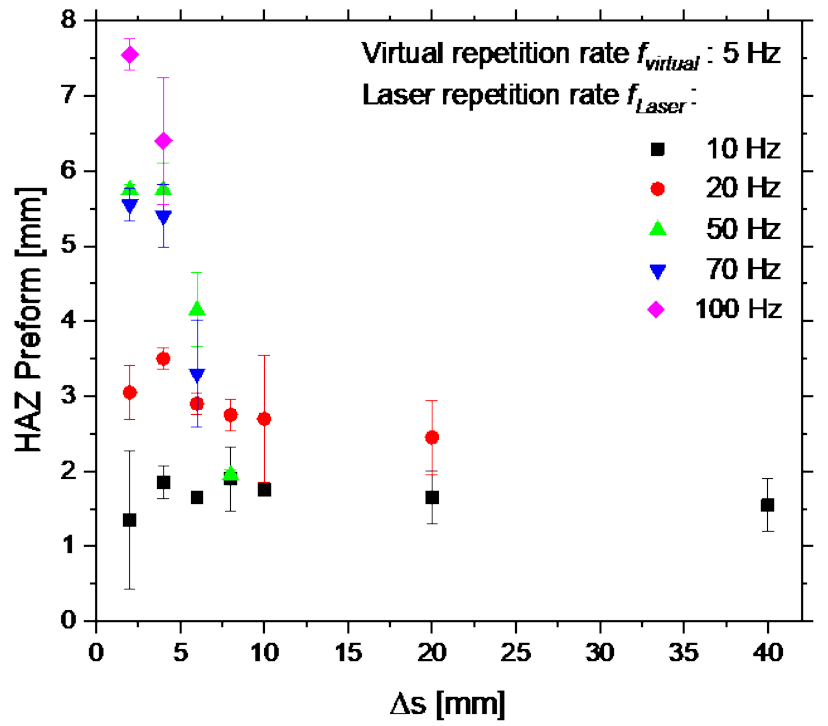

Fig. $8 \mathrm{HAZ}$ at entry for preforms depending on the geometrical pulse distance in one line

Significant temporal-spatial pulse-to-pulse heat accumulation is present for laser repetition rates of $50 \mathrm{~Hz}$ and higher. Thus, the benefit for reducing the process time is quite limited if, at the same time, a minimum HAZ shall be achieved. If a HAZ of more than $2-3 \mathrm{~mm}$ is allowed the benefit for a pulse distribution strategy takes effect.

Demonstration of an advantageous pulse distribution strategy for a straight line cut

The potential of the pulse distribution strategy shall be shown in a case where HAZ is permitted to a certain degree. A fast, low quality (LQ), a slow, high quality (HQ) continuous scan-cut as well as the pulse distribution strategy are compared in Fig. 9. The total length of the line is $84 \mathrm{~mm}$.
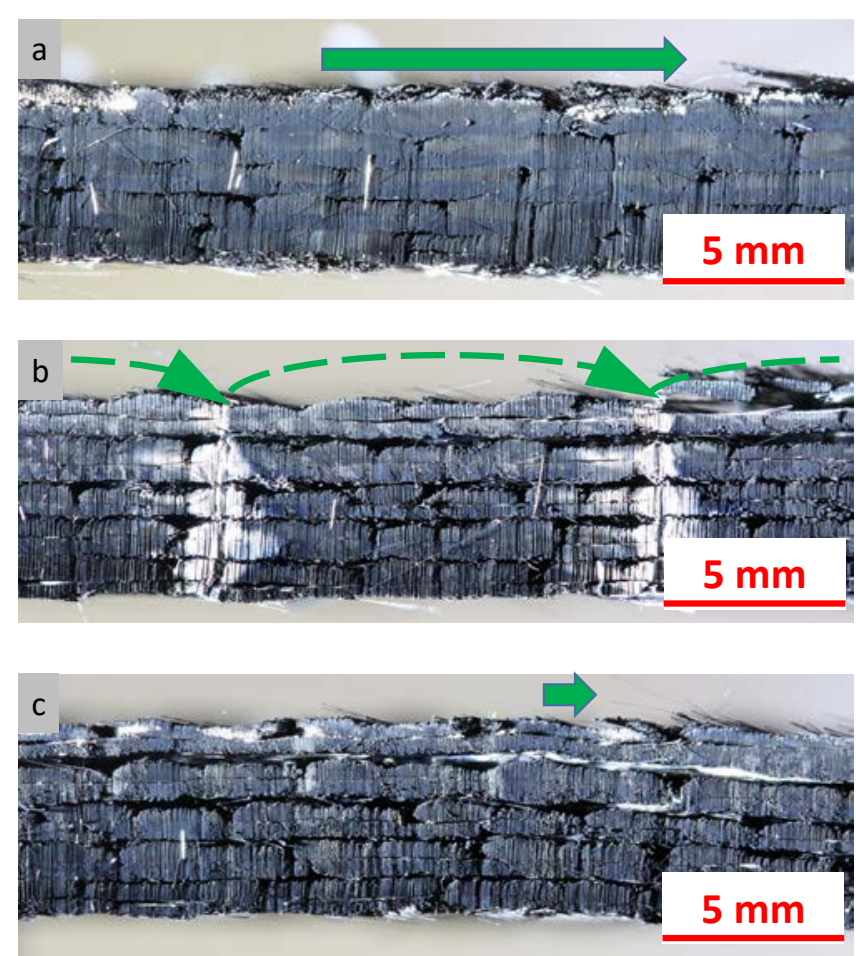

Fig. 9a shows a clearly visible solid layer on top of the fiber ends at the cut wall which is not desired for the subsequent matrix infusion process. Though it might not negatively influence the matrix infusion process itself, the solid layer can tear the vacuum foil in a vacuum infusion process. In a resin transfer molding (RTM) process the upper mold of the tool could break the solid layer and press chunks of broken layer material into the preform which could lead to micro cracks or they could scratch the protective layer of the insert.

The top of the preform in Fig. 9d shows a significant HAZ of about $6 \mathrm{~mm}$. Within the first $3 \mathrm{~mm}$ the graphite structure of the carbon fibers has significantly changed due to the heat input. These areas are very brittle and tend to break more easily than the unharmed fibers. The fibers in the heat affected zone have at least partly lost their sizing which is necessary to increase adhesion forces between the fibers and the matrix. Though the $70 \mathrm{~Hz}$ continuous scancut process is relatively fast, the HAZ is $5.6 \mathrm{~mm}$ wide.

Fig. 9c shows the same process but with a laser repetition rate of $10 \mathrm{~Hz}$ and a smaller scanning speed. A solid layer is still present but it is not as thick and continuous as the layer in Fig. 9a. The HAZ in Fig. 9f is significantly smaller and does not show severe graphitization of the carbon fibers. The size of the HAZ is about $1.8 \mathrm{~mm}$. This process can be regarded as a high-quality process. The small $\mathrm{HAZ}$ and relatively small solid layer at the cut wall are beneficial for a subsequent matrix infusion process. However, the total process time is seven times longer than the fast continuous scanning process.

The benefit of a pulse distribution strategy is shown in Fig. 9b and Fig. 9e. The materialographical quality corresponds to the slow scan-cut results but the productivity is as high as the fast continuous scan-cut process. The grey

\begin{tabular}{|l|l|}
\hline \multicolumn{2}{|l|}{ Continuous scanning (fast, LQ) } \\
\hline Pulse peak power $\hat{P}$ & $3 \mathrm{~kW}$ \\
\hline Pulse duration $t_{p}$ & $0.5 \mathrm{~ms}$ \\
\hline Repetition rate $f_{\text {Laser }}$ & $70 \mathrm{~Hz}$ \\
\hline Scanning speed $v$ & $2.85 \mathrm{~mm} / \mathrm{s}$ \\
\hline Pulse overlap $P O$ & $50 \%$ \\
\hline Process time $t_{\text {proc }}$ & $29.5 \mathrm{~s}$ \\
\hline
\end{tabular}

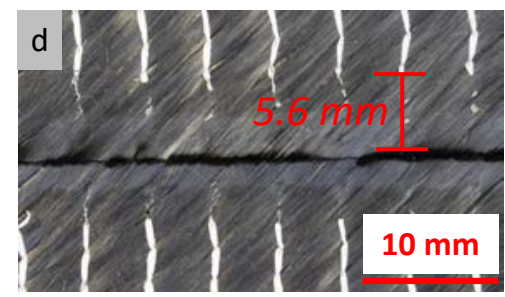

\begin{tabular}{|l|l|}
\hline Pulse distribution strategy (fast, $\mathrm{HQ}$ ) \\
\hline Pulse peak power $\hat{P}$ & $3 \mathrm{~kW}$ \\
\hline Pulse duration $t_{p}$ & $0.5 \mathrm{~ms}$ \\
\hline Repetition rate $f_{\text {Laser }}$ & $70 \mathrm{~Hz}$ \\
\hline Sim. repetition rate $f_{\text {sim }}$ & $10 \mathrm{~Hz}$ \\
\hline Pulse overlap $P O$ & $50 \%$ \\
\hline Process time $t_{\text {proc }}$ & $32 \mathrm{~s}$ \\
\hline
\end{tabular}

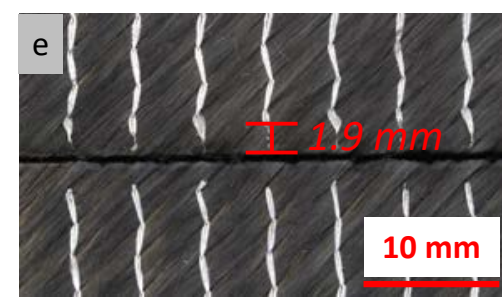

\begin{tabular}{|l|l|}
\hline \multicolumn{2}{|l|}{ Continuous scanning (slow, $\mathrm{HQ}$ ) } \\
\hline Pulse peak power $\hat{P}$ & $3 \mathrm{~kW}$ \\
\hline Pulse duration $t_{p}$ & $0.5 \mathrm{~ms}$ \\
\hline Repetition rate $f_{\text {Laser }}$ & $10 \mathrm{~Hz}$ \\
\hline Scanning speed $v$ & $0.41 \mathrm{~mm} / \mathrm{s}$ \\
\hline Pulse overlap $P O$ & $50 \%$ \\
\hline Process time $t_{\text {proc }}$ & $204.9 \mathrm{~s}$ \\
\hline
\end{tabular}

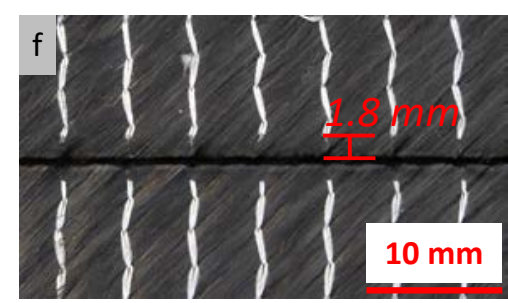

Fig. 9 Cross section (a-c) and HAZ at entry (d-f) of a fast and slow continuous scan-cut compared to a pulse distribution strategy 
vertical lines in Fig. 9b appear at those points where the pulses of the very last line are shot just before the process ends.

In terms of productivity and materialographical quality the pulse distribution process is the most beneficial out of the three shown processes.

Transformation of the strategy to a round hole for force transmission elements (e.g. inserts)

The transformation of the pulse distribution strategy to a round hole is similar to the one for a straight line. One major difference is that the end of one circle line is the beginning of the next circle line. No big jumps from one end of a line to the beginning of a new line are required. Another difference is that the distance $\Delta s$ between pulses in one circle is limited to the diameter of the scan path $d_{\text {scan }}$.

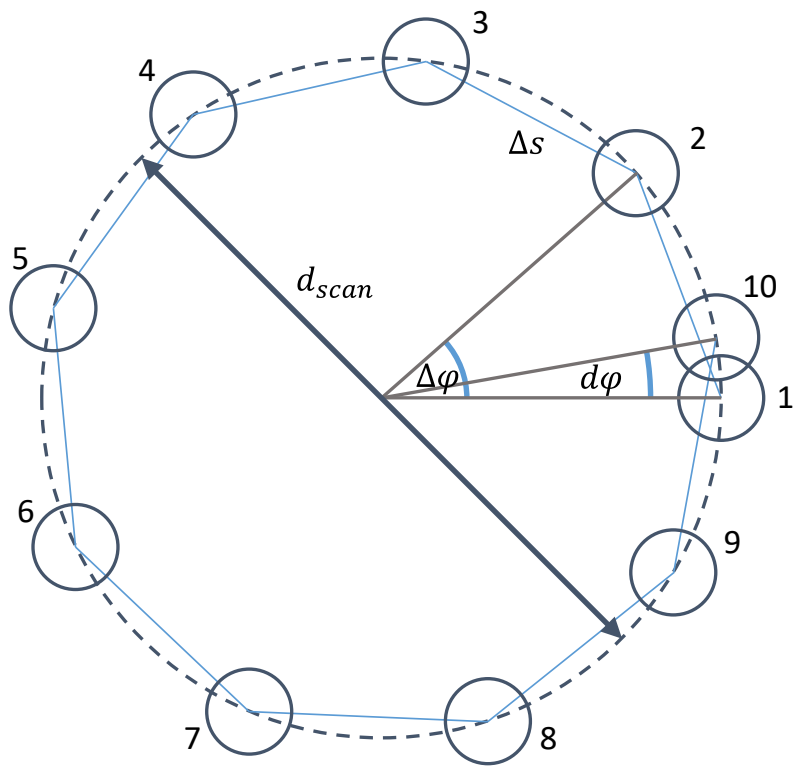

Fig. 10 Schematic pulse distribution strategy for a round hole

The relevant equations for the pulse distribution strategy for a circle are:

Pulse overlap angel in between circle start points$$
d \varphi=2 \cdot \arcsin \left(\frac{d_{f o c} \cdot(1-P O)}{d_{\text {scan }}}\right)
$$

Minimum number of pulses required

$N_{P, \min }=\frac{360^{\circ}}{d \varphi}$

Number of pulses per circle line

$$
N_{P / R}=\left\lceil\frac{f_{\text {Laser }}}{f_{\text {virtual }}}\right\rceil
$$

Distribution angle for pulses in one circle line

$\Delta \varphi=\frac{360^{\circ}+d \varphi}{N_{P / R}}$

Number of circle

lines

$N_{R}=\left\lceil\frac{\Delta \varphi}{d \varphi}\right\rceil$

Total number of pulses

$$
N_{P}=N_{P / R} \cdot N_{R}
$$

True pulse overlap angel

$d \varphi_{\text {true }}=\frac{360^{\circ}}{N_{P}}$
True distribution angle

$$
\begin{aligned}
\Delta \varphi_{\text {true }} & =\frac{360^{\circ}+d \varphi_{\text {true }}}{N_{P / R}} \\
P O_{\text {true }} & =1-\frac{d_{\text {scan }} \cdot \pi}{d_{\text {foc }} \cdot\left(N_{P}-1\right)}
\end{aligned}
$$

True pulse overlap

Linear distance between pulses in one circle

$$
\Delta s=d_{\text {scan }} \cdot \sin \left(\frac{\Delta \varphi}{2}\right)
$$

Total process time $\quad t_{\text {proc }}=\frac{N_{P}}{f_{\text {Laser }}}$

whereat $P O$ in equation 15 is the initial desired pulse overlap. The procedure is analog to the one for a straight line with respect to the limitations and conditions to a circular geometry mentioned earlier. In order to achieve a minimum HAZ the laser repetition rate is even more limited due to its connection to $\Delta s$ (s. equation 13).

In order to show these limitations the process parameters from Fig. 9 have been applied to a circular scanning path with a diameter $d_{\text {scan }}$ of $8.5 \mathrm{~mm}$. The relevant process parameters and the results regarding HAZ are shown in Table 7 and Fig. 11.

Table 7 Process parameter and results for different scanning strategies of a circular scanning contour

\begin{tabular}{cccc} 
& $\begin{array}{c}10 \mathrm{~Hz} \\
\text { continuous } \\
\text { scanning }\end{array}$ & $\begin{array}{c}\text { Pulse } \\
\text { distribution } \\
(70-10 \mathrm{~Hz})\end{array}$ & $\begin{array}{c}70 \mathrm{~Hz} \\
\text { continuous } \\
\text { scanning }\end{array}$ \\
\hline$E_{P}$ & $1.275 \mathrm{~J}$ & $1.275 \mathrm{~J}$ & $1.275 \mathrm{~J}$ \\
$d_{\text {scan }}$ & $8.5 \mathrm{~mm}$ & $8.5 \mathrm{~mm}$ & $8.5 \mathrm{~mm}$ \\
$f_{\text {Laser }}$ & $10 \mathrm{~Hz}$ & $70 \mathrm{~Hz}$ & $70 \mathrm{~Hz}$ \\
$f_{\text {virtual }}$ & - & $10 \mathrm{~Hz}$ & - \\
$P O_{\text {simple }}$ & $49.7 \%$ & $50.08 \%$ & $50.04 \%$ \\
$v_{\text {scan }}$ & $0.41 \mathrm{~mm} / \mathrm{s}$ & - & $2.85 \mathrm{~mm} / \mathrm{s}$ \\
$\Delta s *$ & $(0.041 \mathrm{~mm})$ & $3.66 \mathrm{~mm}$ & $(0.041 \mathrm{~mm})$ \\
$t_{\text {proc }}$ & $64.538 \mathrm{~s}$ & $9.3 \mathrm{~s}$ & $9.3 \mathrm{~s}$ \\
HAZ & 2.12 & 5.41 & 5.35 \\
$($ entry) & & 7.54 & 7.61 \\
HAZ & 4.28 & & \\
$($ exit) & & &
\end{tabular}

* The distance between pulses for both continuous scanning processes is the linear pulse overlap which is half of the focal diameter for $50 \%$ pulse overlap

The results for HAZ at the entry correspond to the results shown in Fig. 8, even though the virtual repetition rare is slightly different. The main reason for this result is the limited distance between subsequent pulses on a circular scanning path ( $\Delta s$ (Fig. 11b): 3.66 mm; $\Delta s$ (Fig. 9b): $12 \mathrm{~mm}$ ). 


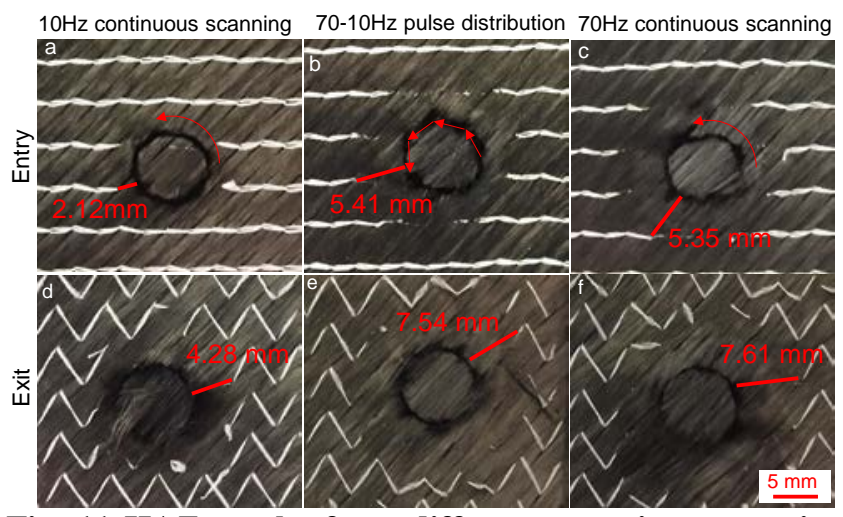

Fig. 11 HAZ results from different scanning strategies for $\emptyset 8.5 \mathrm{~mm}$ circular drilling contours. Arrows indicate scanning resp. jump directions. Drilling cores not yet removed

Hence, the smallest HAZ can only be achieved if the virtual repetition rate is half of the laser repetition rate. Furthermore, the laser repetition rate should be as small as possible for a predefined drilling hole contour. The larger the hole diameter, the higher the laser repetition rate can be set to achieve the same size of HAZ.

\section{Conclusions \& Outlook}

Drilling of carbon fiber preforms by means of long pulsed fiber laser radiation is a productive and mechanically contactless method to manufacture holes for force transmission elements. The main disadvantage of using long pulse laser radiation is the generation of heat which burns away the sizing which is the protective and functional layer on top of the carbon fibers. Additionally, the generation of a thick solid layer at the hole wall can occur. The hard but brittle layer could not only negatively influence the subsequent matrix infusion process but also harm the corrosion protection layer of the insert if the foil in vacuum infusion process or the mold of a RTM machine compresses the preform with a pre-integrated insert.

Even at a low laser repetition rate of $5 \mathrm{~Hz}$ in a continuous scanning process heat accumulation takes place. Due to pure temporal or spatial-temporal pulse-to-pulse heat accumulation the knitting yarn, as an indicator of the HAZ, burns away several millimeters from the hole contour. Since such low laser repetition rates lead to excessive process times, a specially designed pulse distribution strategy has been proposed which has the potential to not only regain productivity but also to ensure geometrical quality in terms of a small HAZ and size of the solid layer. By aid of the pulse distribution process for long pulsed laser radiation introduced in this work, the pulses in a pulse sequence are spread in recurring lines over the drilling contour. The geometrical distance due to a high laser repetition rate between two subsequent pulses in one line must be big enough to avoid heat accumulation. The time between directly overlapping pulses (virtual repetition rate) of each two subsequent lines must be long enough to avoid temporal pulse-to-pulse or, in this particular case, scan-to-scan heat accumulation.

If the design of the hole offers enough space to distribute pulses in one line with sufficient distance a minimum heat affected hole can be expected. In case of a very small hole, approaches to reduce HAZ are either to use a low laser repetition rate or switch to another hole and distribute pulses in between several holes if several holes within the total scan field can be drilled. Due to the required high pulse energy of more than $1.2 \mathrm{~J}$ for one-shot-penetration of five bi-axial layers of carbon fibers and due to the relatively high heat capacity, heat conductivity and heat diffusivity of the carbon fibers, the waiting time in between pulses becomes a crucial factor.

The presented pulse distribution strategy has been developed and evaluated for five layered bi-axial carbon fiber preforms. The achievable results will not only vary for thinner and thicker preforms but also between unidirectional (UD), multidirectional non-crimp fiber (NCF) and woven fabrics. Finally, a broad and deep investigation on the material performance under different mechanical loads is required to further point out the potential benefits of the pulse distribution strategy.

This strategy works well with long pulsed laser radiation and small repetition rates. Pulses can be placed individually and accurately within a pulse pause due the high dynamics of nowadays scanners. The distribution strategy could also be modified and be transferred for a short or ultra-short pulsed laser source. Due to the significantly higher repetition rates, the pulse distribution could be done via pulse bursts in order to mimic a long pulse drilling process.

Further improvements regarding the process time could be the variation of the focal diameter. The bigger the focal diameter, the less pulses are required to drill a macroscopic drilling contour. However, it must be ensured that enough energy and intensity can be applied to evaporate the carbon fibers.

\section{Acknowledgements}

The authors would like to thank Sebastian Oppitz from Institut für Textiltechnik ITA of RWTH Aachen University for the provision of carbon fiber preforms.

\section{References}

[1] A. Riveiro, F. Quintero, F. Lusquinos, J. del Val, R. Comesana, M. Boutinguiza and J. Pou: Composites: Part A, 43, (2012) 1400.

[2] R. Negarestani, L. Li, K. Sezer, D. Whitehead and J. Methven: Int. J. Adv. Manuf. Technol., 49, (2010) 553.

[3] Y. Sato, M. Tsukamoto, F. Matsuoka, T. Ohkubo and N. Abe: Appl. Surf. Sci., 417, (2017) 250.

[4] A. N. Fuchs, M. F. Zäh: wt Werkstatttechnik online, 104, (2014) 394.

[5] C. Cherif: "Textiler Werkstoff für den Leichtbau“, (Springer, Heidelberg, 2011) p.82.

[6] A. Boyle, K. Dunne: European Patent EP1328372B1 (2006)

[7] H. Eickenbusch, O. Krauss: "Kurzanalyse Nr. 3: Kohlenstofffaserverstärkte Kunststoffe im Fahrzeugbau - Ressourceneffizienz und Technologien“, (VDI ZRE, Berlin, 2014) p.13.

[8] P. Mucha, N. Speker, R. Weber, T. Graf: Appl. Phys. A, 113, (2013) 361. 
[9] DIN 32540:2012-08: "Laser beam removing - Thermal removing with laser beam - Terms and definitions, influence factors, procedure"

[10]S. Janssen, A. Gillner, S. Oppitz: Proc. 17th Int. Symp. on Laser Precision Microfabrication, Xi'an, (2016), \#16-30

(Received: June 5, 2017, Accepted: March 27, 2018) 